FUNCTIONS OF A MENTAL HEALTH (MH) TEAM ON A PEDIATRIC ONCOLOGY UNIT. Jesus Nahmias and M. Lois Murphy. Memorial Sloan-Kettering Cancer Center, Department of Pediatrics, New York.

Since 1969,400 patients were seen by the MH Team and 300 had psychometrics. In 1973, 50 were in individual and group play therapy and 50 parents were in group therapy over 6 months. There were over 300 multidisciplinary case presentations. Recurrent themes of emotional abandonment and symbiosis had clinical manifestations of poor or no attendance at school and acting-out behavior at home and in community: isolation, withdrawal, hopelessness, helplessness and regression. The MH Team's goal is prevention and rehabilitation. Clinical research was undertaken to institute and validate methods. Patients' systems were approached in all components (hospital, family, school, community) and followed by the MH nurse. Results are improvement in school attendance, participation in the community, survival of peer contact, improvement of self-esteem, development of trust, less anxiety and less fear. Parents learned acceptance and became active participants in hospital, school, community and in the therapeutic efforts. There was better understanding among other staff of rehabilitation techniques and MH concepts. They developed better systems of communication between patients and parents becoming familiarized with the concept of a therapeutic community. Clinical cases will be presented. Supported by NCI Grant \#CA05826; ACS Pediatric Cancer Psychiatry Program; Acquavella Pediatric Fund \#2160-545.

A MODEL SYSTEM FOR THE PREVENTION AND CONTROL OF CHILD ABUSE: CONCEPTUAL, ETHICAL AND PRACTICAL CONSIDEPATIONS. ELI H. Newberger, Julius B. Richmond, Children's Hospital Medical Center and Judge Baker Guidance Center, Boston, Massachusetts Child abuse can be seen as a phenomenon for which parental dysfunction is the cause; as an artifact of a system of childrearing which encourages violent coercion to behavioral norms and as a tragic expression of a culture which deprives some families of the services and goods of society. A program to prevent and control child abuse would see, measure, and act on each of these dimensions. It would comprehend the ir relative importance in the population and develop policies, evaluative tools and intervention methods to assure that children's and families' health and well-being would be sustained and promoted.

A Governor's Commission on Child Abuse found in a review of 961 case reports and a survey of 281 practitioners the familiar bias of selection for reporting towara poor and minority children and a striking sevenfold ratio of unreported to reported cases. Subsequently, a new program was developed for Massachusetts which attends to recent advances in theory, practice, and law and implements a management.

Newly-passed legislation defines child abuse as a symptom of family crisis. A case registry and hotline provide a wider portal of entry into the existing service structure, and new services attend to local problem-solving methods and childrearing traditions, as defined by citizen-based Councils for Children: The objective of the program is the improvem protection of their of fspring.

GEOGRAPHIC DISTRIBUTION OF LIKE-SEX TWINS IN THE U.S, by George M. Owen, Univ. of New Mexico Sch. of Med. Dept. of Ped., AIbuquerque and Stanley M. Garn, Univ. of Michigan, Ctr. of Human Growth and Development, Ann Arbor.

Between 1968 and 1970, 3441 preschool children were included in a national study of nutritional status based on a probability sample drawn by the Univ. of Michigan Survey Research Center. The purpose of this report is to comment on the geographic distribution of 29 like-sex twin pairs, 17 of which were female-female. From radiographs (motacarpal-phalangeal profile patterns), anthropometry, biochemical determinations and correspondence with families, it appeared that nearly half the like-sex twins were identical. Altogether, $90 \%$ of like-sex twin pairs (26) lived in the northerm half of the country (above latitude $37.5^{\circ}$ ) although only $55 \%$ of the study population lived in the northern half of the country. Further analysis of data suggested some relation between frequency of like-sex twinning and altitude. Only $7.5 \%$ of the study population resided at elevations greater than 900 meters above sea level but it accounted for $40 \%$ ( 12 pairs) of like-sex twins. Four pairs of like-sex twins ( $3 F-F$ and $1 M-M$ ) were encountered in one Califormia county at an elevation of 2000 meters.
THE DETECTION OF SUBCLINICAL Pb INTOXICATION IN URBAN CHILDREN. S. Piomelli, P. Young, A. Brickman, V. Guinee, The upper limit of "normal" blood Pb level (BPb; in $\mu \mathrm{g} /$ $100 \mathrm{ml}$ whole blood) in urban children is undetermined, al though values $\geq 40$ are officially considered abnormal. This study sought to determine the $\mathrm{BPb}$ at which the earliest metabolic effects of $\mathrm{Pb}$ become apparent and whether measurements of Free Erythrocyte Porphyrins (FEP; in $\mu \mathrm{g} / \mathrm{g} \mathrm{Hgb}$ ) are a more sensitive indication of increased body burden of $\mathrm{Pb}$.

The correlation between FEP and BPb was analyzed in 1892 samples of blood submitted for BPb to the NY City Dept. of Health. In the 640 samples with $\mathrm{BPb} 0-30$ there was no correlation; mean FEP was 2.4 , and $8 \%$ had positive FEP test $\left(\mathrm{FEP}^{+}=\mathrm{FEP}>5.5\right)$, presumably due to $\mathrm{Fe}$ deficiency. As $\mathrm{BPb}$ reached 30, FEP levels increased exponentially. In the 297 samples with BPb $30-39$ mean FEP was 3.5 , and $16 \%$ were $\mathrm{FEP}^{+}$. The FEP level rose at the same fast exponential rate up to $\mathrm{BPb} 60$, where mean FEP was 17 and $100 \%$ of the samples were $\mathrm{FEP}^{+}$. At $\mathrm{BPb}>60$, FEP rose further at a slower rate. EDTA provocation was performed in $31 \mathrm{FEP}^{+}$children with $\mathrm{BPb}$ 30-54. Abnormal urine $\mathrm{Pb}$ was found in $7 / 8$ children with $\mathrm{BPb} 30-39$; in $7 / 8$ with $\mathrm{BPb} 40-44$; in $8 / 9$ with $\mathrm{BPb} 45-49$ and in $6 / 6$ with $\mathrm{BPb} 50-54$.

These data indicate that FEP levels increase significantly much before BPb reaches the "official" value of 40 and detect increased body burden of $\mathrm{Pb}$ with greater sensitivity than $\mathrm{BPb}$.

MOTHER TO CHILD SPEECH AT TWO YEARS: THE EFFECTS OF INCREASED EARLY POST-NATAL CONTACT. Norma Ringler, John Kennell, Marshall Klaus, Billie Navojosky. Case Western Reserve Univ. Sch. of Med., Dept. of Ped., Cleveland.

Can obstetric practices influence a mother's speech to her child two years later? This is the third follow-up of a continuing study designed to determine if mother-infant separation during the early post-partum hours results in altered mothering behavior for a prolonged period. 28 primiparous mothers of normal, full-term infants were assigned randomly to one of 2 study groups. The control mothers had routine physical contact with their babies (a glimpse at birth, a brief visit for identification at 6-12 hours and visits for feeding every 4 hours). The extended-contact mothers in addition, had one hour of physical contact with their nude infants in the first 3 hours after birth and then 15 additional hours in the first 3 days.

The speech patterns of 10 of the mothers selected at random from these 2 groups were compared, without knowledge of study groups, as they spoke to their children during a 15 minure unstructured play period at a two-year follow-up visit. Significant differences in mother-to-child speech were noted. Mothers in the extended-contact group used twice as many questions $(p<.05)$, more adjectives $(\alpha<.02)$, more words per proposition $(p<.05)$, fewer commands $(p<.05)$ and content words $(p<.02)$ than the controls. No differences were noted when the mothers spoke to adults. These results suggest that the linguistic environment of the young child may be indelibly shaped by early mother-infant care practices.

\section{COMPUTER SUPPORT FOR MONITORING NORMAL CHILD GROWTH}

\section{Charles P. Schade, Charles L. Moffet, and Carlos Vallibona}

\author{
Baylor College of Medicine \\ Department of Community Medicine \\ Houston, Texas
}

Assessment of children's growth is an important aspect of preventive pediatrics which can be delegated to a nurse practitioner. In order to facilitate the nurse's assessment, we have developed a computer program which calculates the percentile standing of a child with respect to his peers in weight, length/height, or fronto-occipital head circumference (FOC) or any other observed variable of normal growth which can be tabulated for a substantial population of children.

This program is currently being tested in a pediatric outpatient clinic where the children's growth status is incorporated in a computerized outpatient record.

A related program can be used to generate curves for the plotting of normal growth in a new format which displays growth from birth to adolescence on a semilog scale. We propose to use the program online for detecting growth abnormalities in children and for the generation of portions of a computerized pediatric cardiology report. 